\title{
A Stroboscopic Vibration Analyzer
}

\author{
S. Edelman, R. Brooks, S. Saito, E. Jones, and E. R. Smith
}

(July 15, 1959)

\begin{abstract}
An instrument for studying the amplitude and phase relations which exist in complex periodic processes is described in this paper. The process treated here is vibration of complicated bodies. The motion at each point of interest is detected by a vibration pickup. The signal from each pickup is heterodyned with a common signal that differs from the vibration frequency by a small amount to produce a set of signals at the difference frequency. The desired relations are exhibited at the difference frequency which is low enough to give a slow motion effect. The output circuitry allows scope for ingenuity to choose a presentation suited to a particular problem.
\end{abstract}

\section{Introduction}

Stroboscopic instruments have been used for the visualization and analysis of periodic motion for many years. The simple stroboscope, consisting of a light flashing at an adjustable rate, has long been used for studying rotating machinery and low frequency vibrations in the laboratories and industrial plants. More sophisticated designs such as the Chadwick-Helmuth Slip Sync, the Winkler StroboSync, or the International Research and Development Corporation Vibration Analyzer, permit more detailed analysis of the motion by synchronizing the flash of light with the motion being studied, and the first two provide a means of slowly shifting the phase of the flash so that the entire cycle of the motion is visible. In addition to these commercial instruments there are laboratory instruments $[1,2,3,4$, $5,6,7,8]^{1}$ having the same ability to slow as well as to stop apparent motion at any portion of the cycle and also the ability to observe motion of microscopic amplitude. In all these instruments the vibrating object must be observed directly or through some optical system. Where the field of view is large the allowable magnification of the motion is small.

The "strobolyzer" described here carries stroboscopic vibration analysis further by allowing observation of vibrating bodies of any size and complexity as well as motions whose amplitudes may range from submicroscopic to directly visible, and whose frequencies may range from subsonic to ultrasonic.

This improvement is accomplished by observing the pattern of motion depicted by the response of a number of vibration pickups to the vibration, in place of observing the vibrating body itself. The signals from the pickups are processed in such a way that stroboscopic effects such as apparent slow motion or stopped motion at any phase of the vibration cycle can be obtained.

${ }_{1}^{1}$ Figures in brackets indicate the literature references at the end of this paper.

\section{General Construction}

The basic operation of the strobolyzer is shown schematically in figure 1 . A number of vibration pickups are used to detect the motion of the vibrating body. If the body vibrates at some frequency, ( $f$ cps) the signal from each pickup will be at $f$ cps, but, in general, will differ from the signals of the other pickups in amplitude and phase. The number and location of the pickups are chosen so that all significant aspects of the motion are observed. This choice may be made on the basis of an a priori estimate of the vibration pattern and an originally arbitrary distribution may be modified in the light of increasing knowledge as the study progresses. The signal from each pickup is mixed with a reference signal at $(f-\Delta)$ cps where $\Delta$ is usually about 1 cps but can be varied.

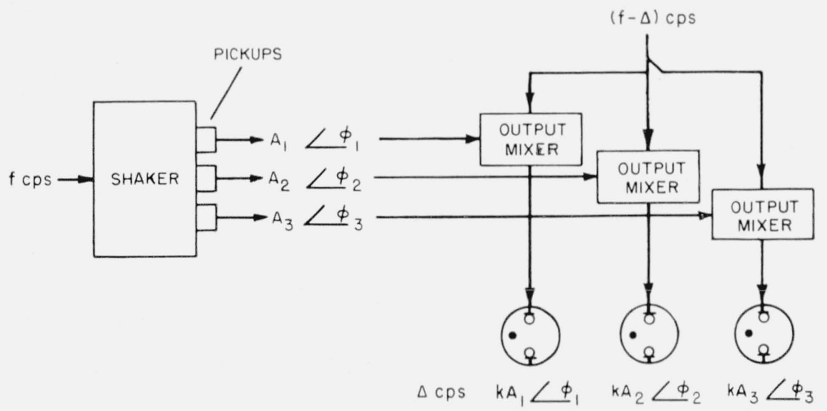

Figure 1. Block diagram of strobolyzer.

If the signal from pickup $i$ is $A_{i} \sin \left(\omega t+\phi_{i}\right)$ and the signal which is mixed with each of the pickup signals is $B \sin (\omega t-2 \pi \Delta t)$, then the signal from the output mixer, $M_{i}$, is the sum of these:

$$
M_{i}=A_{i} \sin \left(\omega t+\phi_{i}\right)+B \sin (\omega t-\delta t),
$$


where $\delta=2 \pi \Delta$,

$$
M_{i}=\sqrt{A_{i}^{2}+B^{2}+2 A_{i} B \cos \left(\delta t+\phi_{i}\right)} \sin \left(\omega t+\Psi_{i}\right)
$$

where $\Psi_{i}=\tan ^{-1}\left(A_{i} \sin \phi_{i}-B \sin \delta t\right) /\left(A_{i} \cos \phi_{i}+\right.$ $B \cos \delta t)$. This is a sine wave at the vibration frequency whose amplitude varies with time according to the expression under the radical sign. This expression can be rewritten as:

$$
B \sqrt{1+r_{i}^{2}}\left\{1+2 \frac{r_{i}}{1+r_{i}^{2}} \cos \left(\delta t+\phi_{i}\right)\right\}^{1 / 2}
$$

where $r_{i}=A_{i} / B$. Expanding by means of the binomial theorem:

$$
\begin{aligned}
M_{i}=B \sqrt{1+r_{i}^{2}} & \left\{1+\frac{r_{i}}{1+r_{i}^{2}} \cos \left(\delta t+\phi_{i}\right)\right. \\
& \left.-1 / 2\left(\frac{r_{i}}{1+r_{i}^{2}}\right)^{2} \cos ^{2}\left(\delta t+\phi_{i}\right)+\ldots\right\}
\end{aligned}
$$

The higher order powers of $\cos \left(\delta t+\phi_{i}\right)$ can be expressed as harmonics of $\delta / 2 \pi$ but if $B>>A_{i}, r_{i}$ will be small and terms containing powers of $r_{i}$ greater than the first are negligible. Since the first term is constant, the time varying part of the amplitude consists of a sine wave whose frequency is $\Delta$. This equation is well known in the theory of heterodyning and is treated in textbooks on radio engineering. It is shown [9] that by making $B$ large compared to $A_{i}$ and by using a square law detector, a signal is obtained at a frequency of $\Delta$ cps whose amplitude is proportional to $A_{i}$ for all practical purposes and whose phase is $\phi_{i}$. A suitable output mixer circuit can be found in electronics texts, for example, Terman [9, p. 569, fig. 41]. The output signal from. each mixer is applied to a suitable indicator, which follows the $\Delta$ cps signal. The group of indicators is arranged approximately in the arrangement of the pickups on the vibrating body. With a little practice the indications are interpreted as motions and the vibration pattern can be visualized. A system somewhat similar to the one treated in this paper was described by Goldman and Richards [10].

\section{Modified Oscillator}

The generation of the signal which differs from the vibration frequency by $\Delta$ cps is shown schematically in figure 2. The vibration frequency ( $f$ cps) is obtained from a General Radio Type $913 \mathrm{C}$ beat frequency oscillator which consists of a fixed oscillator whose signal is always $186,000 \mathrm{cps}$, a variable oscillator whose signal differs from 186,000 cps according to the setting of the tuning dial, a mixer which combines the two signals, and an output section which amplifies the difference of the two signals. In the modification for the strobolyzer a second mixer and output section were placed in parallel with the original sections and a phase shifter was inserted between the fixed oscillator and the new mixer.

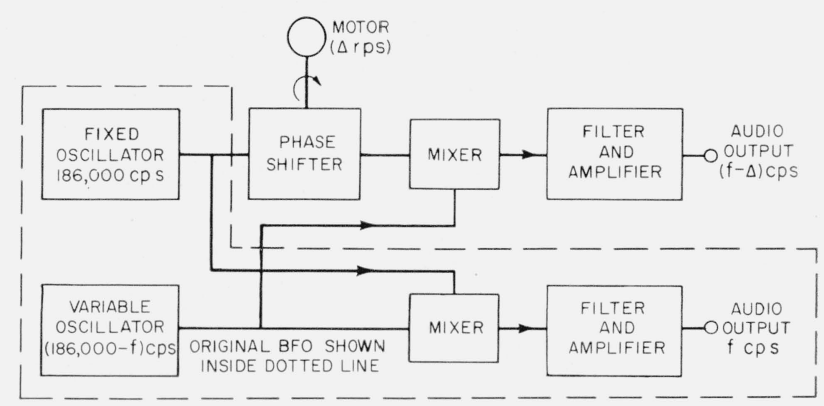

Figure 2. Modified beat frequency oscillator.

The phase shifter used in the modified oscillator is the capacitive type described in Terman's Handbook [9, p. 949, fig. 56e]. When the phase splitting input circuit is properly tuned, the phase shift is equal to the rotation of the shaft in corresponding units and there is no change of amplitude with phase. Since the phase shifter is in the fixed oscillator circuit there is no need to retune the phase splitting circuit for any output frequency of the beat frequency oscillator. If the output of the fixed oscillator is $A_{F}$ $\sin \left(\omega_{F} t\right)$ where $\omega_{F}=2 \pi \times 186,000$ radians per second, the output of the phase shifter will be $C_{\phi} A_{F}$ sin $\left(\omega_{F} t+\theta_{\phi}\right)$ where $C_{\phi}$ comprises the changes in amplitude caused by the insertion of the phase shifter and its associated circuitry while $\theta_{\phi}$ comprises the corresponding phase shifts including the shift due to the initial setting of the phase shifter. $C_{\phi}$ can be made unity by inserting an amplifier ahead of the phase shifter. Constant rotation of the phase shifter at $\Delta \mathrm{rps}$ adds to $\theta_{\phi}$ a phase angle which is a linear function of time: $\theta_{\phi}-2 \pi \Delta t$ and the signal to the mixer is

$$
A_{F} \sin \left(\omega_{F} t+\theta \phi-2 \pi \Delta t\right)=A_{F} \sin \left\{2 \pi(F-\Delta) t+\Theta_{\phi}\right\}
$$

where 186,000 is written as $F$ for convenience. In the original GR mixer, the signal from the fixed oscillator at $F$ cps is mixed with the signal from the variable oscillator at $(F-f)$ cps and the output is the audiofrequency $(f \mathrm{cps})$. In the added mixer the signal from the phase shifter at $(F-\Delta)$ cps is mixed with the signal from the variable oscillator at $(F-f)$ cps and the output differs from $f$ cps by the frequency of rotation of the phase shifter, $\Delta$ cps.

If the phase shifter is not rotated the two outputs will be at the same frequency and the phase difference between them can be set at any value by setting the phase shifter. Thus, if the motor is stopped the indicators of the strobolyzer present the amplitude and phase relations at a particular phase of the vibration cycle. This "freezing" of the vibration pattern corresponds to the stroboscopic "freezing" of the motion when a flashing light is synchronized with the vibration frequency.

If the phase shifter dial is turned slowly by hand, the entire vibration cycle is traversed and can be studied at leisure. Differences in the phase of the motion at different points can be measured by noting the amount of rotation of the phase shifter dial required to move the indicators to corresponding positions. 
If the phase shifter dial is rotated by the motor at $\Delta$ rps the entire vibration cycle is traversed repeatedly at $\Delta$ cps and the indicators show the phase and amplitude relations in slow motion.

\section{Output Presentation}

In the course of developing the strobolyzer a number of methods of output presentation were tried such as blinking neon bulbs and a-c voltmeters. As the number of channels increases it becomes difficult to read or follow a large number of meters or bulbs.

As an alternative a 10 channel bar graph presentation was developed for use with an oscilloscope; this instrument is called the multichannel electronic switch. The basic system samples each channel in succession at a rate sufficiently rapid to accommodate the retentivity of the eye and oscilloscope phosphor.

A Sylvania 6910 decade counter tube provides the sequencing function and counts the output of a 1,500 cps oscillator, shown in figure 3 . Tubes V1 and V2 are used to sequence the counter tube.

The output pulses from the counter tube operate the gates for each channel; QIB is the gate transistor for a typical channel. A negative pulse is required to operate the gate, therefore transistor QIA is used to invert the positive pulses from the counter tube.

Since it is desirable to have a d-c or bar-type presentation instead of a sine wave from the vibration pickups, transistor QIC is used as an emitter follower and $\mathrm{d}$-c rectifier. Transistor $\mathrm{Q}_{0}$ is the output transistor which also serves to invert the negative signals to give them a positive sense.

The oscilloscope $\mathrm{Y}$ axis input is connected to the output of $\mathrm{Q}_{0}$. The $\mathrm{X}$ axis signal is the normal sweep of the oscilloscope itself and is synchronized or triggered from output No. 1 of the counter tube.

In order to accommodate large signals in the order of $40 \mathrm{v}$ rms a divider is used to divide the signal by a factor of 20 .

Occasionally the strobolyzer is used for investigations in which the original display methods are more useful, for example, cases where flashing lights can be arranged to give three dimensional effects. Neon bulbs can be connected to the output channels in such cases, either in conjunction with the oscilloscope display or in place of it.

The output channels also can be connected to recorders. As pointed out by Fred Mintz of Lockheed Aircraft Corp., the fact that the output frequency is always $\Delta$ cps makes it possible to record the significant phase and amplitude relations of vibratory motion at any vibration frequency with recorders of limited dynamic response since $\Delta$ can be chosen to suit the recorder.

Usually the relative amplitudes and phases constitute the desired information. If the absolute value of the motion is desired, the signal from any pickup can be fed into a voltmeter from a $T$ connector at the input to its mixer channel.

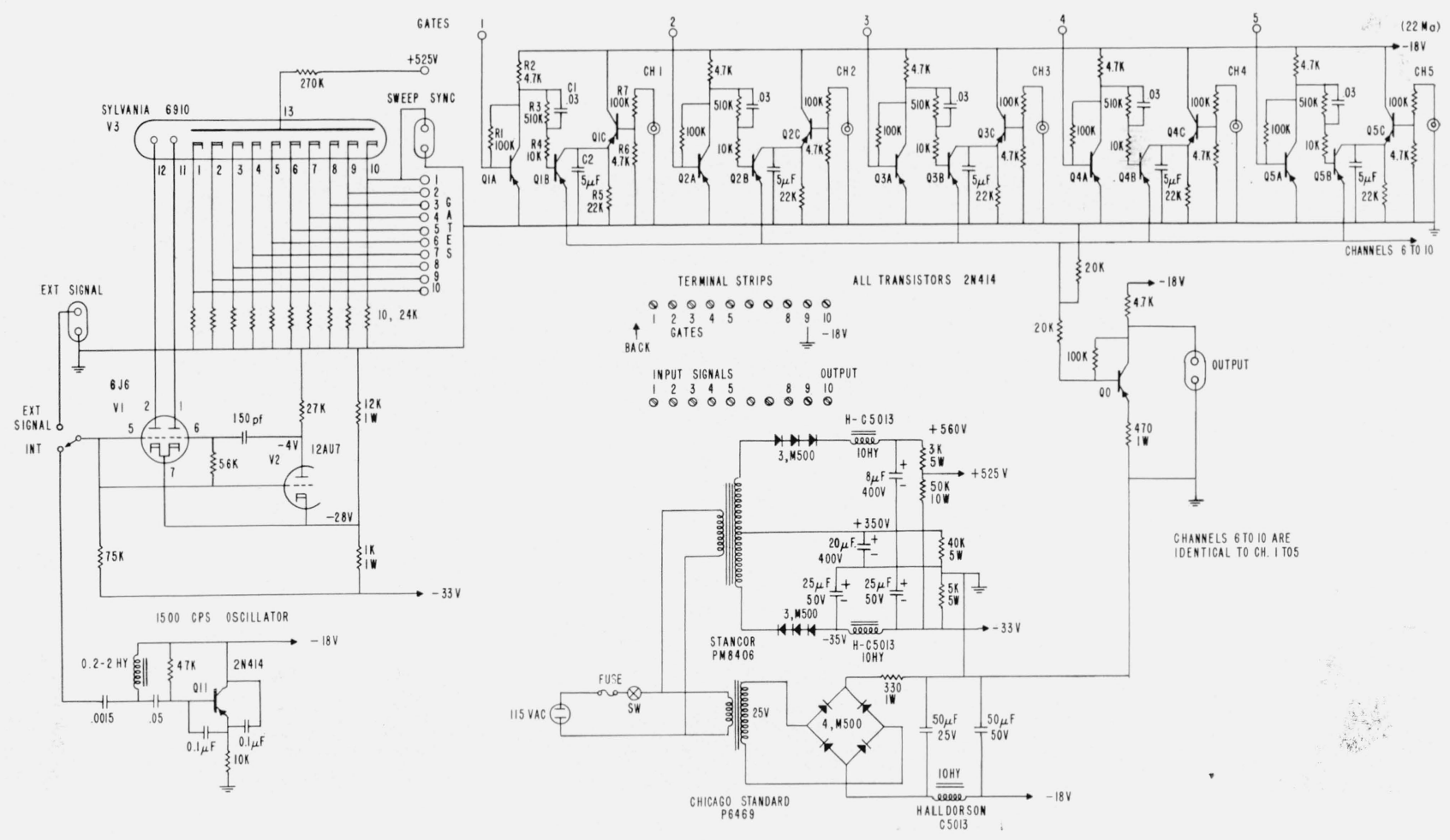

FIGURE 3. Multichannel electronic switch. 


\section{Uses}

The strobolyzer was devised as a tool to aid improvement of vibration exciters for use in calibration of vibration pickups.

The accuracy of such a calibration depends greatly on the use of undistorted uniaxial sinusoidal motion. As techniques of vibration measurement improved it became apparent that a significant amount of distorted motion occurred in the shakers at some frequencies. In order to achieve accurate calibration at these frequencies it was necessary to determine the cause of the unwanted components of the motion; e.g., the excitation of a transverse or flexural mode of some part of the structure. By cementing a number of small, light vibration pickups to all parts of the shaker and by using the strobolyzer to determine the phase and amplitude of motion at each point relative to the other points it was possible to identify the origin of the unwanted motion. A chang of design to minimize such motion usually was no too difficult after the motion was known.

Figure 4a was contrived to illustrate the use of the strobolyzer to study the vibration of a bar excited near the middle by the loudspeaker pot in the foreground. The acceleration was detected by barium titanate pickups, the relative phase of the acceleration at each point being indicated by its corresponding d-c meter. Acceleration phases at four intervals separated by $90^{\circ}$ are shown in figure $4 \mathrm{~b}$.

Figure 5 shows the use of the strobolyzer in studying the pattern of motion at the resonant frequencies of a thin-walled cylinder rigidly fastened at the base. The cylinder walls were too thin to allow mechanical driving or detection of the motion. The vibration was generated acoustically by the loudspeaker which is shown lifted several inches above its usual position in driving the structure. The mo-

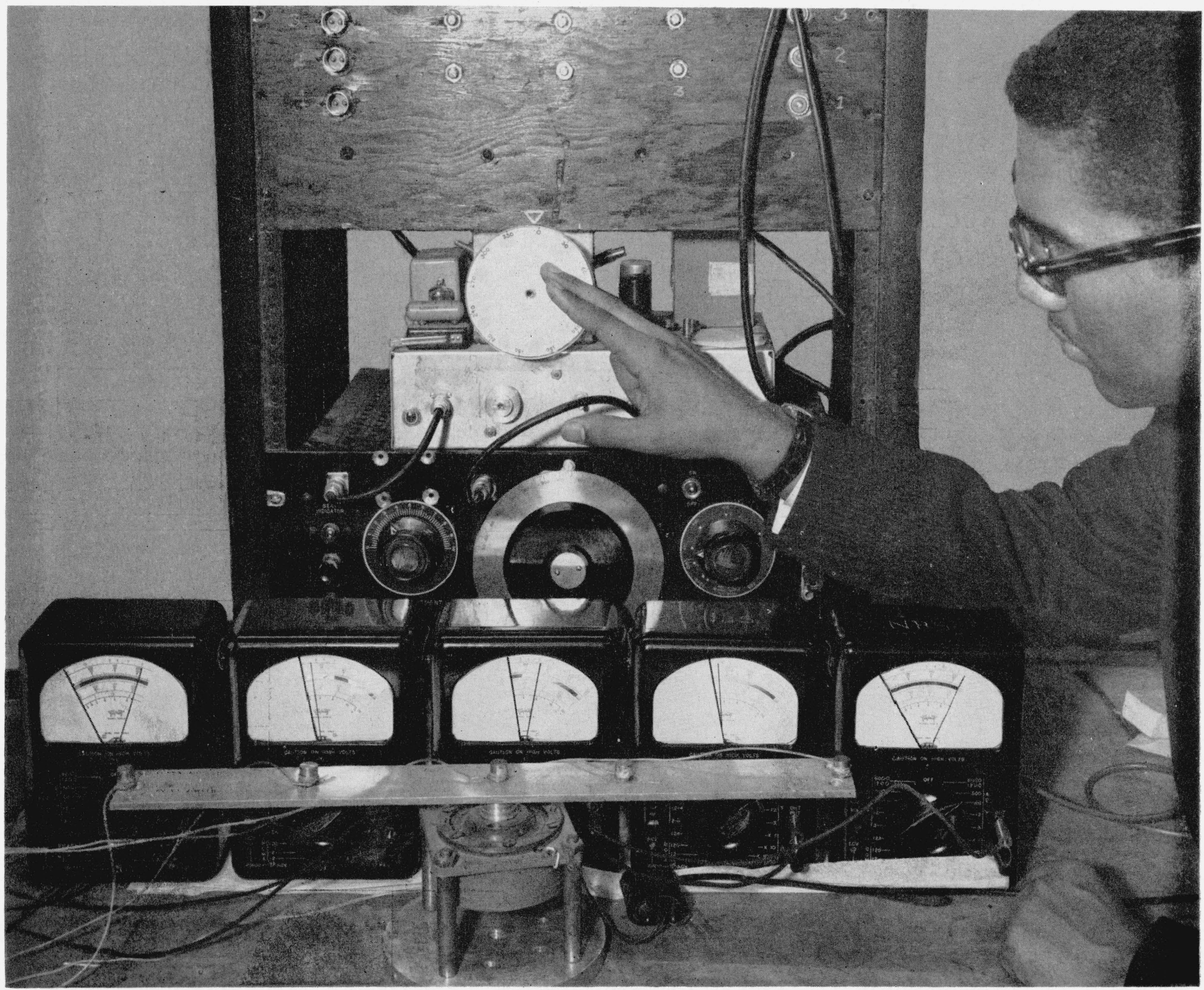

FIGURE 4a. Acceleration of a bar driven at the center.

Meter scales have been masked to show limits of indicator excursion as phase shifter is turned through $360^{\circ}$. Unmasked scale angle is proportional to peak acceleration. 

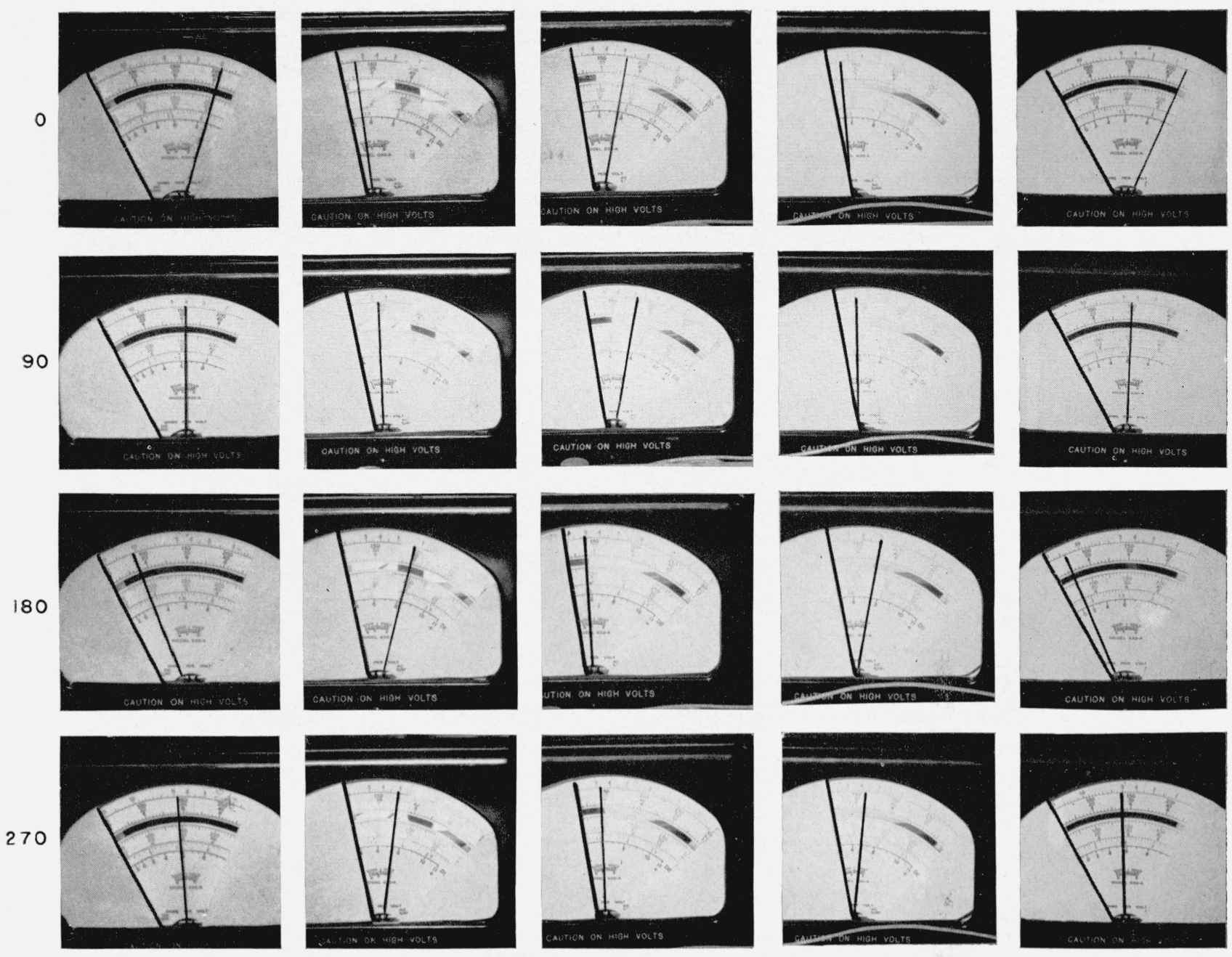

FIGURE 4b. Acceleration of a bar driven at the center.

Indicator positions give relative phase of acceleration at each pickup position for phase of vibration cycle shown on phase shifter dial.

tion of the cylinder walls was detected by noncontacting capacitive vibration pickups whose design was adapted from that described by Shafer and Plunkett [11]. The bases of the pickup supports were constructed so that the spacing between the cylinder wall and the pickup did not change as the pickup was moved around the periphery. Two of the pickup supports carried two pickups each to detect standing waves in the axial direction. Since the top of the cylinder was always a loop and the bottom always a node of displacement, a small spread in the position of the lower pickup sufficed to determine the presence of axial waves. The circumferential standing wave pattern was determined by moving the single pickup around the cylinder, noting the drop in amplitude and change in phase as a node was passed. Two barium titanate vibration pickups were cemented to the base to detect any motion which would be contrary to the assumption that the base support was rigid. A microphone monitored the sound output to detect any shifts in the pattern of standing waves of sound in the room. In preliminary experiments with the loudspeaker at a considerable distance above the cylinder it was found that movements of reflecting surfaces in the room, such as a change in the position of the observer might shift the whole vibration pattern along the periphery of the cylinder. This was annoying when it occurred part way through the determination of the vibration pattern at a particular frequency. The microphone was introduced to warn of such shifts. Later, it was found that the recurrence of such shifts was reduced by lowering the loudspeaker until it nearly touched the cylinder but they still occurred frequently enough to warrant continued use of the microphone.

The relationships existing among the different signals were displayed on the oscilloscope. The output signals at intervals of approximately $90^{\circ}$ are shown below the picture. The signal indicated 


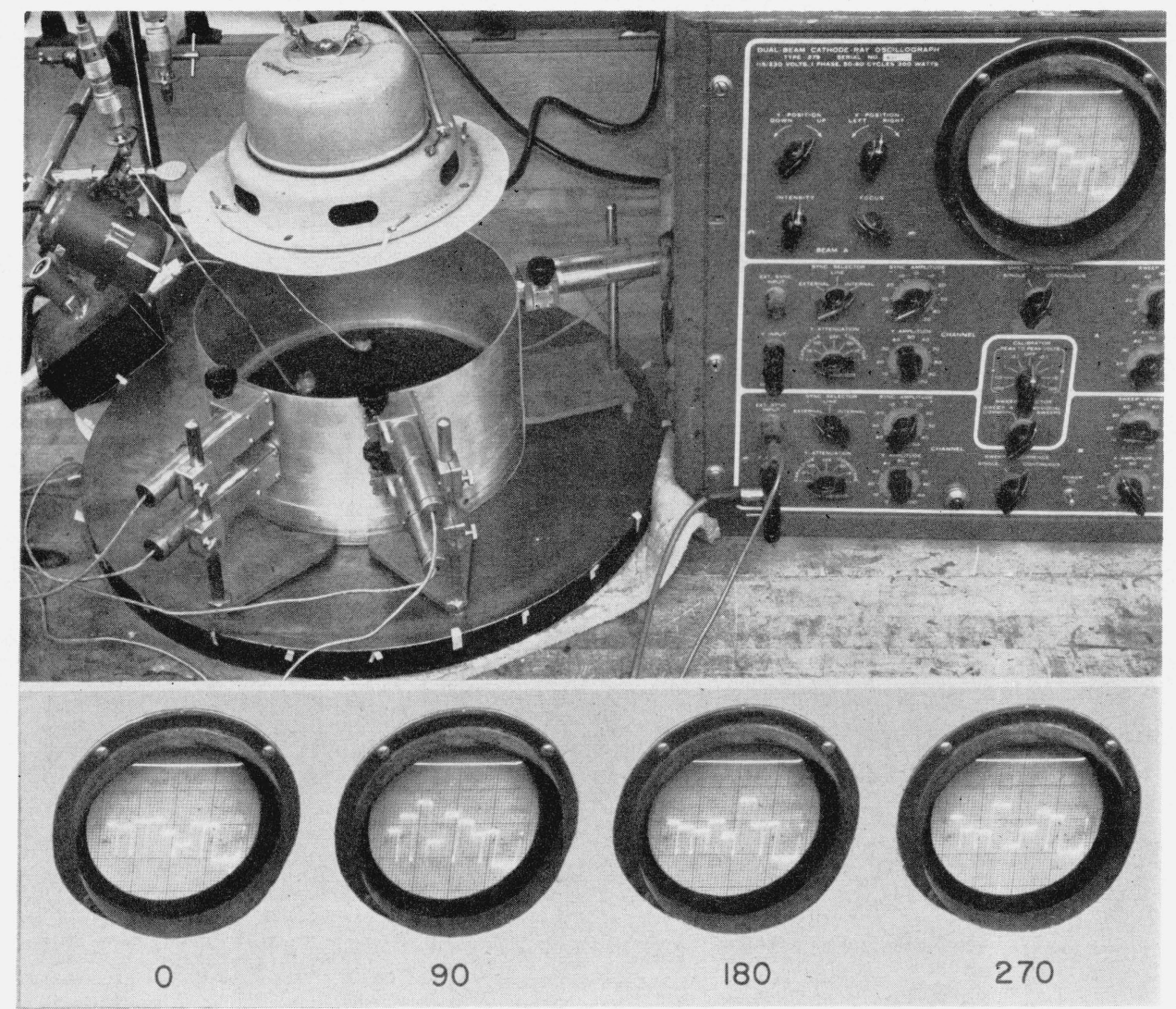

FIGURE 5. Study of resonances of thin walled cylinder.

Height of each bar of the oscilloscope trace gives relative phase and amplitude of corresponding pickup signal at a particular phase of the vibration cycle. The relations among the pickup signals at phase intervals of $90^{\circ}$ of the vibration cycle are displayed below the picture.

by each bar is given in the list below with the bars numbered from left to right.

Signals Indicated by Oscilloscope Display

\begin{tabular}{ll} 
Bar & \multicolumn{1}{c}{ Signal from pickup } \\
1 & left lower capacitive pickup \\
2 & right lower capacitive pickup \\
3 & left upper capacitive pickup \\
4 & right upper capacitive pickup \\
5 & mobile capacitive pickup \\
6 & loudspeaker signal used as a reference for phase \\
7 & measurement \\
8 & mierophone signal
\end{tabular}

As the examples described above indicate, the strobolyzer is more useful as an exploratory tool than as a routine measuring device. If the information of interest is the amplitude of vibration at a number of points for the purpose of estimating damage or malfunction, the strobolyzer has no advantage over conventional methods. However, if the problem is to visualize the varied details of the motion of a complex structure or to determine interactions among various motions, the strobolyzer provides unique assistance.
Since it is a new instrument, its effectiveness in probing problems depends to some extent on the ingenuity of the user in taking full advantage of its flexibility. For example, the motion to be studied need not be excited by the modified beat frequency oscillator shown in figure 2 and may not be under the control of the experimenter. In this case, the signal from one of the pickups is taken as the reference. The reference signal is applied through the phase shifter to each of the inner channels as shown in figure 6 so that the output of each mixer channel is at $\Delta$ cps. When the strobolyzer is used in this way it is necessary to tune the phase shifter to each audiofrequency which is used. One convenient way to do the tuning is to use ganged wafer switches to connect matched pairs of resistors and capacitors for selected frequencies which are used frequently and to use trimmer capacitors for intermediate frequencies. Considerable information can be obtained by proper filtering of the pickup signals.

The strobolyzer is flexible in the wide variety of transducers which may be used to supply the input signals, and therefore also in the wide variety of periodic phenomena which may be studied. 


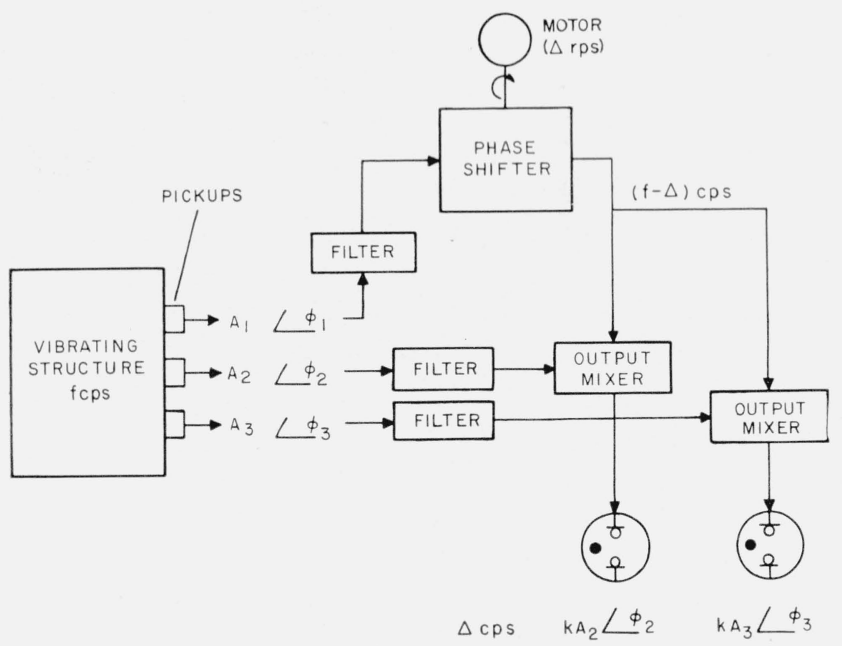

Figure 6. Use of strobolyzer to study externally excited motion.

Sidney Kohrn of the Bureau staff helped considerably in the development and early use of the instrument described here.

In the course of several stimulating discussions, G. F. Sheets, Jr. of the White Sands Proving Ground contributed to the development of the output circuit.

\section{References}

[1] Paul Paasche, Z. tech. Physik. 9, 411 (1928)

[2] W. D. Dye, Proc. Roy. Soc. (London) A138, 1 (1932).

[3] G. F. Hull, Rev. Sci. Instr. 15, 340 (1944).

[4] Bruce, Macinante, and Kelly, Nature 167, 520 (Mar. 31, 1951).

[5] S. Tolansky and W. Bardsley, Proc. Phys. Soc. (London) B64, 224 (1951).

[6] Bruce, Macinante, and Kelly Australian J. App. Sci. 4, 28 (1953).

[7] E. A. G. Shaw, J. Acoust. Soc. Am. 28, 38 (1956).

[8] E. R. Smith, S. Edelman, E. Jones, and V. A. Schmidt, J. Acoust. Soc. Am. 30, 867 (1958).

[9] F. E. Terman, Radio Engineers Handbook, McGrawHill Book Co., Inc., p. 567, (1943).

[10] D. E. Goldman and J. R. Richards, Measurement of High Frequency Sound Velocity in Mammalian Soft Tissues, J. Acoust. Soc. Am. 26, p. 981, (Nov. 1954).

[11] S. N. Shafer and R. Plunkett, Proc. Soc. Exp. Stress Anal., XIII, No. 1, p. 123 (1955).

Washington, D.C.

(Paper 63C2-12) 